\title{
The Effects of Aromatherapy, Meditation, and Blue Light on Sleep Quality
}

\author{
Amy Potter ${ }^{1}$ \\ ${ }^{1}$ St. Paul's School, United States of America \\ Correspondence: Amy Potter, St. Paul’s School, United States of America. E-mail: amy.potter@sps.edu
}

Received: February 1, 2019

Accepted: February 27, 2019

Online Published: May 30, 2019

doi:10.5539/mas.v13n5p144

URL: https://doi.org/10.5539/mas.v13n5p144

\begin{abstract}
Sleep insomnia, which reduces the effectiveness of sleep, is a large problem in America that affects individuals with a range of ages. Nearly 60 million people suffer from insomnia in America every year. My goal was to see if the use of aromatherapy, reduction of blue light, and practice of meditation before bed would increase sleep quality. More specifically, I will be focused on increasing the time spent in deep sleep and reducing the times one wakes up during the night. To test my hypothesis, I used the Fitbit Versa to monitor the sleep patterns of the participants of three people with and then the same three people without the pre-bed routines that may affect their sleep. While the results showed that aromatherapy meditation did improve the sleep of the participants, allowing the participant to sleep without waking up as many times are normal and feel more refreshed upon waking, the reduction of blue light had no significant impacts on the participant's quality of sleep.
\end{abstract}

Keywords: meditation, and blue light on sleep quality

\section{Introduction and Prior Works}

60 million people suffer from insomnia in America every year (Valentine). While scientists and sleep specialists disagree on the best treatment for insomnia, they agree that it is a growing issue (Valentine). Research shows that teenagers with insomnia often exhibit excessive daytime sleepiness which increases the risk of poorer academic performances, increased irritability, depressive moods, and more car accidents (Bootzin). Similarly, adults experience both immediate and longer-term consequences including impaired memory, a decrease in alertness and performance, and a larger number of psychiatric disorders (Bootzin). Overall, sleep insomnia, which reduces the effectiveness of sleep, is a large problem in America that affects individuals with a range of ages.

The effectiveness of sleep can be measured in many different ways. First, sleep can be measured in terms of quantity (ie: the hours of sleep one has experienced). In general, the longer time someone sleeps, the better they will feel in the morning, regardless of any pre-bed routines or in sleep devices. Second, sleep can be measured in terms of its regularity. Sleep regularity refers to the consistency of someone's bedtime and hours of sleep as well as their sleep cycles ("How Is Sleep Quality Calculated?"). Third, the quality of the sleep can be measured. Affected by different interventions and pre-bed routines, sleep quality looks at the time spent in the deep sleep and the number of times someone wakes up during the night. Therefore, we can improve the quality of sleep by increasing the time spent in deep sleep while reducing the number of times one wakes up. To better understand deep sleep, we must first understand what the different stages of sleep are. Every night, our bodies go through several sleep stages - light, REM, and deep- which last for roughly 90 minutes each. The common sleep cycle begins with light sleep; as the entry into sleep each night, the body uses this time to wind and slow down. During light sleep, people may drift between falling asleep and being awake, making the body somewhat alert and easily woken. Light sleep promotes the mental and physical recovery needed by the body. Next, the body falls into deep sleep. Generally occurring within the first hour of sleep, deep sleep promotes physical recovery, improves memory, and supports the immune system. The body becomes less responsive to outside stimulation and harder to wake up. Deep sleep is often used to measure sleep quality as it makes people feel the most refreshed the next day ("What Should I Know about Sleep Stages?"). The body returns to light sleep before entering the REM stage. REM, rapid eye movement, is usually the time when one is dreaming as the brain becomes more active. REM promotes mood regulation, learning, and memory by collecting and consolidating information from the previous day to store in long-term memory ("What Happens When You Sleep?"). The body then repeats this process, falling back into light sleep. 
In a recent study, David S. Black and his colleagues show that the practice of mindful awareness (MAPS) can improve sleep disturbances by looking at the differences between the two groups using a sleep quality assessment called PSQI. While one group of participants used MAPS, the second group tried sleep hygiene intervention, or SHE. Participants in the MAPS group had a mean PSQI of 10.2 (17) at the baseline and improved to a PSQI of 7.4 (1.9) post-intervention (Black). This establishes that MAPS is a reliable method to improve sleep quality. While this study looks at the difference between MAPS and SHE, I will just be comparing MAPS and two specific elements of SHE, aromatherapy, and reduction of blue light, all of which can be done before bed.

Lavender oil, a type of aromatherapy, has been proven to increase sleep quality in a recent study by the British Association of Critical Care Nurses. The experiment gave participants $2 \%$ lavender oil via inhalation for fifteen days. At the end of the fifteen days, the study found that the lavender oil had increased the quality of sleep and reduced anxiety among the participants (Karadag). This shows that lavender oil can be used to increase sleep quality. My experiment differed from this study because it focused specifically on looking at the effect of aromatherapy on patients in the ICU.

Lastly, I tested the effects of reducing blue light before bed. Blue light, a color visible to the human eye, is a short wavelength which produces high amounts of energy. According to an article published by Harvard Health Publishing, "light interferes with a person's circadian rhythm and melatonin secretion". A separate experiment designed by Harvard researchers showed that a shift in the use of blue light can suppress melatonin for twice as long as green light (Harvard Health Publishing). This proves that blue light can decrease the quality of sleep. While this experiment compared blue light and green light, my experiment only looks at the blue light.

\section{Methods}

In my experiment, I focused on meditation, aromatherapy, and the reduction of blue light. My goal was to see if the use of aromatherapy, reduction of blue light, and practice of meditation before bed would increase sleep quality. More specifically, I will be focused on increasing the time spent in deep sleep and reducing the times one wakes up during the night. To test my hypothesis that the use of aromatherapy, reduction of blue light, and practice of meditation before bed does increase sleep quality, I monitored the sleep patterns of three people with and then the same three people without the pre-bed routines that may affect their sleep. Although these sleep cycles are traditionally measured in labs using electroencephalogram to detect brain activity, eye, and muscle movement, new technology allows people to track their sleep cycles at home ("What Should I Know about Sleep Stages?"). In my experiment, I choose to use the Fitbit Versa to track the sleep patterns of the participants. The Fitbit Versa uses wrist actigraphy, autodetection of movement, length of movements, and heart rate, to calculate the time a person spends in each stage of sleep as well as the number of times awoken ("What Should I Know about Sleep Stages?"). Wrist actigraphy has been proven to be able to estimate sleep duration and wakefulness on an individual level (Marino). In research by the Study of Osteoporotic Fractures Research Group, researchers found that, when comparing actigraphy and polysomnography (a sleep study test that records brain waves, the oxygen level in blood, heart rate and breathing, and eye and leg movements), there was no significant difference between the two. In comparison to the polysomnography, actigraphy had an average overestimation of 6.7 minutes and a correlation of 0.58 (Blackwell). This proves that the method I used to collect data is relatively accurate and therefore can have conclusions drawn from it. Participants also completed a survey where they rated how rested they felt on a scale of 1 (not rested) to 10 (very rested). I also took note of other possible factors such as diet and exercise. After providing data from the Fitbit for at least ten days of normal sleep patterns, each participant was randomly selected to test one of the following interventions: aromatherapy, reduction of blue light, and practice of meditation before bed for ten consecutive days. Participants testing aromatherapy used a lavender oil spray and lotion before bed. Participants testing the reduction of blue light stopped the use of blue light thirty minutes before bed light and only did activities without blue light such as reading or journaling. Lastly, participants testing meditation practiced mindfulness meditation for fifteen minutes before bed. All of the collected data was analyzed by comparing the mean percent of time spent in deep sleep, mean number of times woken up, and the mean survey responses for participants before and after the intervention.

\section{Results}

Participant 1 tested aromatherapy for ten days. Participant 1 had never tried using aromatherapy before bed previous to the experiment. While data from Participant 1 shows that there was a decrease in the percentage of time spent in deep sleep, these findings were not significant. However, the increase in survey rating and times awoke during the night was significant. On a normal night, the participant rated their sleep at 4.9 which increased to 6.1 when using aromatherapy ( Fig. 1). Additionally, On a normal night, the participant awoke 29 times on average, but the mean decreased to 21.4 when using aromatherapy ( Fig. 1). This shows that aromatherapy does 
improve the sleep of participant 1, allowing the participant to sleep without waking up as many times are normal and feel more refreshed upon waking.

\begin{tabular}{llll}
\hline Variable & Mean \% of Deep sleep & Mean \# of times woken & Personal Rating \\
\hline None & 15.2 & 29 & 4.9 \\
Aromatherapy & 14.6 & 21.4 & 6.1 \\
\hline
\end{tabular}

Figure 1. Participant 1 Aromatherapy data

Participant 1 then tested meditation for ten days right after the ten days of testing aromatherapy. Participant 1 had never practiced mindfulness meditation previous to the experiment. While data from Participant 1 shows that there was a decrease in the number of times woken throughout the night and the percentage of time spent in deep sleep, these findings were not significant. However, the increase in survey rating was significant. On a normal night, the participant rated their sleep at 4.9 which increased to 6.25 when meditating before bed (Fig. 2). While the participant may not have experienced higher quality sleep when meditating, as shown by the insignificant changes of times woken and percentage of deep sleep, the participant may have felt a placebo effect. Overall, meditation is still a sleep intervention that improves the sleep of participant 1 because the participant believed that they had received better sleep and woke up feeling more energized than normal, regardless of how well they actually slept.

\begin{tabular}{llll}
\hline Variable & Mean \% of Deep sleep & Mean \# of times woken & Personal Rating \\
\hline None & 15.2 & 29 & 4.9 \\
Meditation & 16.5 & 30 & 6.25 \\
\hline
\end{tabular}

Figure 2. Participant 1 Meditation data

Participant 2 tested meditation for ten days. Participant 2 had never practiced mindfulness meditation previous to the experiment. While data from Participant 2 shows that there was a decrease in the percentage of time spent in deep sleep and personal rating, these findings were not significant. However, the increase in times awoken during the night was significant. On a normal night, the participant awoke 26 times on average, but the mean decreased to 19 when using meditation (Fig. 3). This shows that while meditation does improve certain aspects of sleep for Participant 2, the participant did not feel any changes. Participant 2 should try other interventions that may improve sleep quality more for this participant.

\begin{tabular}{llll}
\hline Variable & Mean \% of Deep sleep & Mean \# of times woken & Personal Rating \\
\hline None & 19.25 & 26 & 6.14 \\
Meditation & 22 & 19 & 5.9 \\
\hline
\end{tabular}

Figure 3. Participant 2 Meditation data

Participant 3 tested the reduction of blue light for ten days. Participant 3 had never reduced blue light before bed previous to the experiment. Participant 3 tested the effects of reducing blue light before bed. While data from Participant 3 shows that there was a decrease in the percentage of time spent in deep sleep and personal rating, these findings were not significant. However, the increase in times awoken during the night was significant. On a normal night, the participant awoke 27.1 times on average, but the mean decreased to 21.6 when using reducing blue light (Fig. 4). This shows that while reducing blue light before bed does improve certain aspects of sleep for Participant 3, the participant did not feel any significant changes. Participant 3 should try other interventions that may improve sleep quality more for this participant. 


\begin{tabular}{llll}
\hline Variable & Mean \% of Deep sleep & Mean \# of times woken & Personal Rating \\
\hline None & 15.1 & 27.1 & 6.75 \\
Blue light & 14 & 21.6 & 5.75 \\
\hline
\end{tabular}

Figure 4. Participant 3 Blue light data

\section{Discussion}

In addition to the data results, participants also reported changes or lack of changes that they felt. When looking at the data, aromatherapy only seemed to decrease the number of times participants were awoken throughout the night; however, Participant 1 reported feeling more relaxed the next morning and throughout the day, regardless of how much sleep they received. It was also reported that they fell asleep faster and felt more relaxed when going to bed. This is in agreement with previous research that showed the positive effects of aromatherapy on sleep. Meditation seemed to increase the time spent in deep sleep, helping Participant 1 spend more time in deep sleep and aiding the participant in falling asleep faster. Although she did not feel more rested immediately upon waking, the participant noted that she felt more awake and rested throughout the rest of day and had longer lasting energy. On the other hand, Participant 2 did not feel any significant changes while meditating before bed. This adds to previous research by showing that a sleep intervention may work better for one person than another. Lastly, although the reduction of blue light appeared to decrease the number of times woken up, Participant 3 did not notice any significant changes in how refreshed he felt in the morning as a direct result of the reduction of blue light. This shows some difference from previous research that argued the effectiveness of the reduction of blue light on improving sleep quality.

In addition to the three interventions I chose to focus on (aromatherapy, reduction of blue light, and the practice of meditation), it is important to consider the role of potential confounding variables. Dreams, working out, waking without an alarm clock, drinking tea, watching movies, and eating a light dinner have all been shown to increase sleep quality. Working late, drinking beer, dehydration, hot temperatures, naps, and mosquitos can decrease sleep quality. Additionally, the participants were subject to the placebo effect, an effect which can lead participants to believe that a treatment works regardless of the treatments results. All these factors may have contributed to the results found in this research study.

\section{Future Works}

In the future, I would do this experiment with more participants and over a longer period of time. I think this would provide more accurate results to support my findings. I would also compare sleep quality and quantity to the grades that students get in school. I think it would be interesting to see if any of the variables that I tested would help students get better sleep and the effects of better sleep on their grades. Lastly, I would like to see if there was any correlation between whether the participant had any dreams and the quality of sleep. Using my findings, I could make an app that people could use to track their daily patterns and that could suggest an intervention that may help users improve their sleep quality. I believe that my findings from the future research proposed would be able to help many people across the world sleep better at night. A better night of rest may lead to an improvement in mental health, academic, and work performance.

\section{References}

Bootzin, Richard R., \& Dana, R. E. (2011). Understanding and Treating Insomnia. Understanding and Treating Insomnia, 3. https://doi.org/10.1146/annurev.clinpsy.3.022806.091516

Fernandez, C., et al. (2017). 0067 Computational Phenotyping In Polysomnography: Using Interpretable Physiology-Based Machine Learning Models To Predict Health Outcomes. Sleep, 40. https://doi.org/10.1093/sleepj/zsx050.066

Harvard Health Publishing. (n/a). Blue Light Has a Dark Side - Harvard Health. Harvard Health Blog. Retrieved from http://www.health.harvard.edu/staying-healthy/blue-light-has-a-dark-side

“How Is Sleep Quality Calculated?" Sleep Cycle. Retrieved from http://support.sleepcycle.com/hc/enus/articles/206704659-How-is-Sleep-Quality-calculated-

Karadag, E., et al. (2015). Effects of Aromatherapy on Sleep Quality and Anxiety of Patients. Nursing in Critical Care, 22(2), 105-112. https://doi.org/10.1111/nicc.12198

Marino, M., et al. (2013). Measuring Sleep: Accuracy, Sensitivity, and Specificity of Wrist Actigraphy Compared to Polysomnography. Sleep, 36(11), 1747-1755. https://doi.org/10.5665/sleep.3142 
Valentine, V. (2008). “Can't Sleep? Neither Can 60 Million Other Americans.” NPR, NPR, 20 May 2008. Retrieved from http://www.npr.org/templates/story/story.php?storyId=90638364

"What Happens When You Sleep?" National Sleep Foundation, sleepfoundation.org/how-sleep-works/whathappens-when-you-sleep

Blackwell, Terri, et al. (Oct. 2008). Comparison of Sleep Parameters from Actigraphy and Polysomnography in Older Women: The SOF Study. Sleep, 31(2), 283-291. https://doi.org/10.1093/sleep/31.2.283

Black, D. S., et al. (Jan. 2015). Mindfulness Meditation and Improvement in Sleep Quality and Daytime Impairment Among Older Adults With Sleep Disturbances. JAMA Internal Medicine, 175(4), 494. https://doi.org/10.1001/jamainternmed.2014.80

"What Should I Know about Sleep Stages?" Fitbit Help, 6 July 2018, Retrieved from http://help.fitbit.com/articles/en_US/Help_article/2163/?1=en_US\&c=Topics:Sleep\&fs=Search\&pn=1

Martin, J. L., \& Alex, D. H. (2008). “Wrist Actigraphy.” 28 Nov. 2010. https://doi.org/10.1378/chest.10-1872

\section{Copyrights}

Copyright for this article is retained by the author(s), with first publication rights granted to the journal.

This is an open-access article distributed under the terms and conditions of the Creative Commons Attribution license (http://creativecommons.org/licenses/by/4.0/). 